\title{
An LMI Approach to Optimal Consensus Seeking in Multi-Agent Systems
}

\author{
E. Semsar-Kazerooni and K. Khorasani
}

\begin{abstract}
In this paper an optimal control design strategy to guarantee consensus achievement in a multi-agent network is developed. Minimization of a global cost function for the entire network guarantees a stable consensus with an optimal control effort. In solving the optimization problem it is shown that the solution of the Riccati equation cannot guarantee the consensus achievement. Therefore, the linear matrix inequality (LMI) formulation is used to solve the corresponding optimization problem and simultaneously to address the consensus achievement constraint. Moreover, using the LMI formulation a controller specific structure based on the neighboring sets can be imposed as an additional LMI constraint. Therefore, the only information each controller needs is the one it receives from its associated neighbors in its neighboring set. The global cost function formulation provides more insight into the optimal performance of the entire network and would result in a "global" optimal (or suboptimal) solution. Simulation results are presented to illustrate the performance of the multi-agent team in achieving consensus.
\end{abstract}

\section{INTRODUCTION}

There are many advantages for deploying an autonomous network of agents. For instance, enhanced group robustness to individual failures, increased and improved instrument sensing and resolution, reduced cost of operation, and adaptive reconfigurability capabilities have been discussed in [1]. Some applications that necessitate development of these systems are in satellite deployment for distributed Earth or deep space observations; maneuvers of a group of unmanned aerial vehicles (UAVs) for intelligence, surveillance, and reconnaissance (ISR) missions; automated factories; unmanned underwater vehicles (UUVs) for search and rescue; and teams of mobile robots deployed in a hazardous environment where human involvement is dangerous. In order to fully take advantage of these large-scale networks and systems of systems, several prerequisites are required to be satisfied. Some of these are development of reliable communication, optimal power consumption management, and team cooperation as discussed in [2]. These issues are still open areas of research.

Cooperation in a network of multi-agents has received extensive attention in the past several years. The cooperative control may address one of the following issues, i.e. swarm analysis and flocking [3]-[4], rendezvous problem [5], formation control [6], agreement and consensus seeking [7][9]. Consensus seeking has been investigated from different

E. Semsar-Kazerooni is a PhD student at Electrical and Computer Engineering, Concordia University, 1455 de Maisonneuve Blvd. W., Montreal, Quebec, H3G 1M8, Canada e_semsar@ece.concordia.ca

$\mathrm{K}$. Khorasani is with the Department of Electrical and Computer Engineering, Concordia University, 1455 de Maisonneuve Blvd. W., Montreal, Quebec, H3G 1M8, Canada kash@ece.concordia.ca perspectives and with different assumptions. In [7], as one of the pioneer work in this area, linear and nonlinear consensus protocols are suggested for a network of dynamic agents with integrator dynamics and undirected information flow graphs. The relationship between the maximum tolerable time delay in the network and connectivity of the network is defined.

Most of the above mentioned work on consensus seeking have basically focused on only analysis and not explicitly on the design of a control strategy to guarantee consensus achievement. Moreover, in most of the above articles the suggested solutions are restricted to agents with a specific dynamical equation, i.e. an integrator model. However, in the present work the consensus protocols are designed formally and based on the well-known control design techniques for a general linear model of agents.

There are some results in the literature that address designbased consensus protocols, e.g. [10]-[14]. The author in [10] introduced a method based on passivity for solving the coordination problem. An optimal approach to consensus problem is considered in [11]-[13]. In order to solve the team optimal problem, the authors in [11] have assumed that in evaluating the minimum value of each individual cost, the state of other agents are constant. The works in [12], [13] avoid this restrictive assumption by decomposing the control input of individual agents into local and global components. In [14], the dynamics of the entire network are decomposed into two components, namely one in the consensus space and the other in the orthogonal subspace. A set of LMIs are then used to guarantee the stability and consensus achievement using an $\mathrm{H}_{2}$ design strategy.

In the present work, a consensus protocol is "designed" using optimal control and LMI design tools. For this purpose, the idea of decomposing the state vector into two components as introduced in [14] is adopted for solving the optimal consensus problem. As opposed to [14], where the robust performance is analyzed using $\mathrm{H}_{2}$ design methodology, here we start with a Hamilton-Jacobi-Bellman equation. Then, we will use the LMI formulation of the LQR problem. After decomposing the state vector, a global cost function is suggested for the entire network to achieve a stable consensus. The advantage of introducing a global cost function as opposed to the individual cost functions is that the former can provide a better insight into the overall performance of the network. It is shown that the LMI optimization provides more flexibility when compared to the method based on solution of the Riccati equation. The optimal solution that is obtained incorporates all the imposed constraints and suggests a global optimal (suboptimal) solution. Moreover, since the optimal 
control is a multi-objective framework and with the help of the LMI formulation, the proposed method has the advantage of being capable of addressing additional specifications, e.g. limited control input availability, the specific control structure, and consensus achievement constraint.

The organization of the paper is as follows: In Section 2 , the problem definition is formally given. In Section 3, consensus problem is transformed into a stabilizing problem using a state decomposition technique. Application of optimal control theory for consensus seeking is presented in Section 4. Finally, simulation results and conclusions are discussed in Sections 5 and 6, respectively.

\section{PROBLEM DEFINITION}

\section{A. Model Description}

Multi-agent teams: Assume a set of agents $\Omega=\{i=$ $1, \ldots, N\}$, where $N$ is the number of agents and each member of the team has a dynamical representation as governed by:

$$
\begin{gathered}
\dot{X}^{i}=A^{i} X^{i}+B^{i} u^{i}, X^{i} \in R^{n}, u^{i} \in R^{m}, i=1, \ldots, N \\
Y^{i}=c^{i} X^{i}, \quad Y^{i} \in R^{q}
\end{gathered}
$$

Information structures and neighboring sets: In order to ensure cooperation and coordination among team members, each member has to know the status (output) of other members, and therefore members have to communicate with each other. For a given agent $i$, the set of agents connected to it via communication links is called a neighboring set $N^{i}$ :

$$
\forall i=1, \ldots, N, \quad N^{i}=\{j=1, \ldots, N \mid(i, j) \in E\}
$$

where $E$ is the edge set that corresponds to the underlying graph of the network. Here, we assume that the network connections are bidirectional (undirected graph).

Model of interaction between the team members: Assume that the dynamical model of each agent is given by (1) and (2). This model defines an isolated agent of the team, but in reality the agents have some interactions through the information flows that exist among the neighboring agents. In [13], it is shown that each member's dynamics can be described by the following model in which the interaction terms are incorporated:

$$
\begin{gathered}
\dot{X}^{i}=A^{i} X^{i}+B^{i}\left(u_{l}^{i}+u_{g}^{i}\right) \\
u_{g}^{i}=B^{i} \sum_{j \in N^{i}} F^{i j} Y^{j}, Y^{i}=C^{i} X^{i}
\end{gathered}
$$

in which $u_{g}^{i}$ is the interaction term, and $F^{i j}$ is the interaction coefficient which ensures the compatibility of matrices dimensions in the agents' input and output channels.

We can rewrite the dynamical representation of the entire network as follows:

$$
\dot{X}=A X+B U, \quad Y=C X
$$

in which $X, Y$ and $U$ are the entire team state, output and input vectors, respectively, which are the concatenation of all the state and input vectors and are given by:
$X=\left[\begin{array}{lll}\left(X^{1}\right)^{T} & \ldots & \left(X^{N}\right)^{T}\end{array}\right]^{T}, U=\left[\begin{array}{lll}\left(u_{l}^{1}\right)^{T} & \ldots & \left(u_{l}^{N}\right)^{T}\end{array}\right]^{T}$, $Y=\left[\begin{array}{lll}\left(Y^{1}\right)^{T} & \ldots & \left(Y^{N}\right)^{T}\end{array}\right]^{T}$.

Matrices $A, B$ and $C$ are defined as follows:

$$
\begin{aligned}
A & =\left[\begin{array}{l}
A^{1}, 0, \ldots, B^{1} F^{1 j} C^{j}, \ldots, 0 \\
\vdots \\
0, \ldots, B^{N} F^{N j} C^{j}, \ldots, 0, A^{N}
\end{array}\right], \\
B & =\operatorname{Diag}\left\{B^{1}, \ldots, B^{N}\right\}, C=\operatorname{Diag}\left\{C^{1}, \ldots, C^{N}\right\}
\end{aligned}
$$

The terms $B^{i} F^{i j} C^{j}$ represent the interactions that exist among the agents and is modelled as a component in the input channel of each agent.

\section{B. Problem Statement: Consensus in a Team of Multi-Agents}

Our main goal is to ensure agents' state, e.g. velocity, converge to the same value, i.e. $\forall i, j X^{i} \rightarrow X^{j}$. In other words, we desire that the team reaches to a consensus in the subspace spanned by the vector $\mathbf{1}$, that is:

$X_{s s}=\left[\left(X^{1}\right)_{s s}^{T} \ldots\left(X^{N}\right)_{s s}^{T}\right]^{T}=\left[\begin{array}{llll}1 & 1 & \ldots & 1\end{array}\right]^{T} \otimes \omega_{s s}=\mathbf{1} \otimes \omega_{s s}$ where $\omega_{s s}$ is the final state vector to which the states of all agents converge.

Definition 1 (Consensus to $\mathcal{S}$ ): Let $S$ be an orthonormal matrix in $R^{N n \times 1}$. The system (6) achieves consensus to the subspace $\mathcal{S}=\operatorname{span}\{S\}$ if $\mathcal{S}$ is a minimal set such that for any initial condition the state $X(t)$ converges to a point in $\mathcal{S}[14]$.

In the present work we assume that the desired consensus subspace $\mathcal{S}$ is spanned by the unity vector, i.e. $S=\mathbf{1}$.

\section{STATE DECOMPOSITION}

Using the above definition, the orthonormal basis for the subspace $\mathcal{S}$ is denoted by $S_{N n \times 1}=\mathbf{1}$. The orthonormal complement of this matrix is denoted by $\bar{S}_{N n \times(N n-1)}$, which is a basis for the corresponding subspace orthonormal to $\mathcal{S}$. The following relationships are satisfied by these matrices:

$$
\bar{S}^{*} S=0, \bar{S}^{*} \bar{S}=I, S^{*} S=1, \bar{S} \bar{S}^{*}+S S^{*}=I
$$

where $A^{*}$ stands for the conjugate transpose of $A$. Now, the state vector $X$ can be decomposed into two orthogonal components in the above mentioned subspaces and can be written as [14]:

$$
X=\left[\begin{array}{ll}
\bar{S} & S
\end{array}\right]\left[\begin{array}{c}
X_{\bar{s}} \\
X_{s}
\end{array}\right]
$$

Assuming that the control input has a state feedback structure, i.e. $U=K X$, then the dynamical equation of the system would be transformed into:

$$
\left[\begin{array}{c}
\dot{X}_{\bar{s}} \\
\dot{X}_{s}
\end{array}\right]=\left[\begin{array}{c}
\bar{S}^{*} \\
S^{*}
\end{array}\right](A+B K)[\bar{S} S]\left[\begin{array}{c}
X_{\bar{s}} \\
X_{s}
\end{array}\right]
$$

This follows from the fact that $[\bar{S} S]^{-1}=\left[\begin{array}{c}\bar{S}^{*} \\ S^{*}\end{array}\right]$. 
Since our goal is to ensure consensus in subspace $\mathcal{S}$ for the closed-loop system, the following equilibrium condition is imposed on the above dynamical equation [14]:

$$
(A+B K) S=0
$$

In other words the equilibria should lie in the consensus subspace. This condition should be incorporated in the design procedure. Therefore, we will have:

$$
\begin{aligned}
& {\left[\begin{array}{c}
\dot{X}_{\bar{s}} \\
\dot{X}_{s}
\end{array}\right]=\left[\begin{array}{c}
\bar{S}^{*} \\
S^{*}
\end{array}\right](A+B K) \bar{S} X_{\bar{s}}=} \\
& {\left[\begin{array}{cc}
\bar{S}^{*}(A+B K) \bar{S} & 0 \\
S^{*}(A+B K) \bar{S} & 0
\end{array}\right]\left[\begin{array}{c}
X_{\bar{s}} \\
X_{s}
\end{array}\right]}
\end{aligned}
$$

In order to achieve consensus the final state of the system should be a vector in subspace $\mathcal{S}$. Therefore, the component $X_{\bar{s}}$ should converge to zero in steady state. This implies that this part of the system dynamics should be asymptotically stable. Moreover, the dynamics corresponding to $X_{s}$ is only dependent on $X_{\bar{s}}$, and therefore we are only concerned with the dynamics corresponding to $X_{\bar{s}}$ as governed by:

$$
\begin{aligned}
& \dot{X}_{\bar{s}}=\bar{S}^{*}(A+B K) \bar{S} X_{\bar{s}}=\bar{S}^{*} A \bar{S} X_{\bar{s}}+\bar{S}^{*} B K \bar{S} X_{\bar{s}} \\
& =\bar{A} X_{\bar{s}}+\bar{B} \bar{K} X_{\bar{s}}=\bar{A} X_{\bar{s}}+\bar{B} \bar{U}
\end{aligned}
$$

where $\bar{A}=\bar{S}^{*} A \bar{S}, \bar{B}=\bar{S}^{*} B, \bar{K}=K \bar{S}$. If this part of the dynamics is stabilized asymptotically to zero, $X_{s}$ will approach to a constant value which is in the consensus subspace. Therefore, the consensus would be achieved. Now we may design a state feedback control strategy to guarantee the consensus achievement by the closed-loop system. Towards this end, optimal control techniques will be used below to design a controller to guarantee a stable consensus in an optimal manner.

\section{OPTIMAL CONSENSUS SEEKING}

As discussed in the previous section the purpose of the control design is to stabilize that part of the system dynamics which corresponds to the subspace $\overline{\mathcal{S}}=\operatorname{span}\{\bar{S}\}$. Therefore, our goal is to design the corresponding control gain, i.e. $\bar{K}$. Based on this value of $\bar{K}$ the corresponding value of $K$ for the original system can be obtained. Therefore, optimality here refers to the situation where the dynamics of $X_{\bar{s}}$ is stabilized in an optimal manner.

For characterizing optimality we need to define a formal performance index. We can define either individual performance indices or a single index (cost function) for the entire team. The authors have previously proposed individual cost functions and suggested a semi-decentralized control strategy for minimizing these cost functions in [13]. Although the individual cost functions do better fit within a decentralized control structure, they cannot be utilized as an index of the team performance. In contrast, the team cost function which we use here is a good index of the team performance and its minimization can result in a globally optimal (or suboptimal) solution. However, the solution would be centralized. Fortunately, using the LMI formulation, we will show that this centralized solution can be avoided by adding a constraint on the structure of the controller gain matrix.

Now, let us define the team cost function to be minimized as follows:

$$
d=\int_{0}^{\infty}\left\{X_{\bar{s}}^{T} \hat{Q} X_{\bar{s}}+\bar{U}^{T} R \bar{U}\right\} d t, X=[\bar{S} S]\left[\begin{array}{c}
X_{\bar{s}} \\
X_{s}
\end{array}\right](14)
$$

where $\hat{Q}$ has a predefined structure as $\hat{Q}=\bar{S}^{*} Q \bar{S}>0$ (since $\operatorname{rank}\left(\bar{S}_{(N n) \times(N n-1)}\right)=N n-1$, then $\hat{Q}$ would be a PD matrix if $Q_{N n \times N n}$ is selected to be a PD matrix.

In the following subsection, we show that in general the solution of the Riccati equation does not result in a consensus for a network of agents with general dynamical representation. In other words, the linear quadratic regulator (LQR) formulation cannot be used for consensus achievement. Therefore, in the second subsection an LMI formulation is utilized for the optimization problem which can incorporate the requirements of consensus achievement and results in an optimal consensus algorithm.

\section{A. Discussion on the Solution of Riccati Equation}

The problem of minimizing the cost function (14) subject to the dynamical constraint (13) is a standard LQR problem. The solution to this LQR problem can be achieved by solving the corresponding Riccati equation as follows:

$$
\bar{U}=-R^{-1} \bar{B}^{*} P X_{\bar{S}}
$$

where $P$ satisfies the following Riccati equation

$$
P \bar{A}+\bar{A}^{*} P-P \bar{B} R^{-1} B^{*} P+\hat{Q}=0
$$

Therefore, $\bar{K}=K \bar{S}=-R^{-1} \bar{B}^{*} P$ and from the properties of matrix $\bar{S}$ we can find $K$ as $K=-R^{-1} \bar{B}^{*} P \bar{S}^{*}$. Hence, the control input is given by:

$$
U=-R^{-1} \bar{B}^{*} P \bar{S}^{*} X
$$

By applying this input to system (6), the closed-loop dynamics can be written as:

$$
\dot{X}=\left(A-B R^{-1} \bar{B}^{*} P \bar{S}^{*}\right) X
$$

In order to achieve consensus for the closed-loop system, the matrix $S$ should be in the null-space of the closed-loop matrix, i.e. $\left[A-B R^{-1} \bar{B}^{*} P \bar{S}^{*}\right] S=0$. However, the second part of this expression is zero due to the properties of $\bar{S}$ as stated in (8), i.e.

$$
-B R^{-1} \bar{B}^{*} P \bar{S}^{*} S=0
$$

In other words, we should have $A S=0$ to guarantee a stable consensus. The above discussion is formally summarized in the following lemma.

Lemma 1 Consider a team of agents with the team entire dynamics given in (6) and assume that a state decomposition procedure is performed and the consensus seeking problem is reduced to stabilization of the dynamical equation (13). Then, the solution of the corresponding Riccati equation given in (16) which minimizes the cost function (14) subject to the dynamical constraint (13) may not result in a stable 
consensus algorithm unless the consensus subspace is in the null space of the open-loop matrix $A$, i.e.

$$
A S=0
$$

In other words, this solution may not in general provide a stable equilibria in the consensus subspace.

Proof: Follows from the previous constructive derivations.

In general, the condition $A S=0$ may not be satisfied by the subsystems in the network. The above observation may be explained by the fact that according to the definition of $U$, the control only provides a component in $\overline{\mathcal{S}}$. Hence, the term $B U$ does not contribute to the $\mathcal{S}$ component of $\dot{X}$. Therefore, to have a stable solution where $\dot{X}=0$, the term $A X$ should enjoy the same property, i.e. the component of $A X$ in $\mathcal{S}$ subspace should be zero:

$$
A S=0
$$

From the above discussion we can conclude that for a general case the optimal solution obtained by solving the Riccati equation does not guarantee consensus achievement. Therefore, the consensus condition in general should be imposed onto the optimal solution that is achieved through the solution of the Riccati equation as an extra constraint. Hence, the LQR formulation is not appropriate for the purpose of consensus seeking. In the following subsection we try to find an optimal solution for the above minimization problem subject to the consensus constraint.

\section{B. LMI Formulation of the Optimal Consensus Seeking}

As discussed in the previous subsection, the problem of minimizing the cost function (14) subject to the dynamical constraint (13) cannot be solved as a standard LQR problem. Instead, we use the well known LMI formulation for solving the optimal problem. In [15] it was shown that the LQR problem can be formulated as a minimization problem that is constrained to a set of LMIs. Using this formulation, the controller $\bar{U}=\bar{K} X_{\bar{s}}$ that minimizes the cost function (14) is achieved by solving for and determining the appropriate matrix $P$, namely

$$
\begin{aligned}
& \min X_{\bar{s}}(0)^{T} P X_{\bar{s}}(0) \quad \text { s.t. } \\
& P(\bar{A}+\bar{B} \bar{K})+(\bar{A}+\bar{B} \bar{K})^{*} P+\hat{Q}+\bar{K}^{*} R \bar{K} \leq 0
\end{aligned}
$$

where $\bar{K}=-R^{-1} \bar{B}^{*} P$ yields the optimal solution. In [16] it is shown that if instead of the cost function (14) its expected value is considered and certain assumptions on the initial conditions of the system are imposed, the above minimization problem reduces to:

$$
\begin{aligned}
& \min \operatorname{trace}(P) \quad \text { s.t. } \\
& P(\bar{A}+\bar{B} \bar{K})+(\bar{A}+\bar{B} \bar{K})^{*} P+\hat{Q}+\bar{K}^{*} R \bar{K} \leq 0
\end{aligned}
$$

For the dynamical system (13) the inequality constraint (23) can be written as:

$$
\begin{aligned}
& P\left(\bar{S}^{*} A \bar{S}+\bar{S}^{*} B K \bar{S}\right)+\left(\bar{S}^{*} A \bar{S}+\bar{S}^{*} B K \bar{S}\right)^{*} P \\
& +\bar{S}^{*} Q \bar{S}+\bar{S}^{*} K^{*} R K \bar{S} \leq 0
\end{aligned}
$$

By multiplying both sides of this inequality by $P^{-1}$ we get:

$$
\begin{aligned}
& \bar{S}^{*}(A+B K) \bar{S} P^{-1}+P^{-1} \bar{S}^{*}\left(A^{*}+K^{*} B^{*}\right) \bar{S}+ \\
& P^{-1} \bar{S}^{*} Q \bar{S} P^{-1}+P^{-1} \bar{S}^{*} K^{*} R K \bar{S} P^{-1} \leq 0
\end{aligned}
$$

Now define a new variable $Z>0$ that satisfies the following equation [14]:

$$
Z=\bar{S} \bar{S}^{*} Z \bar{S} \bar{S}^{*}+S S^{*} Z S S^{*}
$$

an example of which can be in the following form [14]:

$$
Z=\left[\begin{array}{ll}
\bar{S} & S
\end{array}\right]\left[\begin{array}{ll}
P^{-1} & 0 \\
0 & M
\end{array}\right]\left[\begin{array}{l}
\bar{S}^{*} \\
S^{*}
\end{array}\right]
$$

where both $M=S^{*} Z S$ and $P^{-1}=\bar{S}^{*} Z \bar{S}$ are PD matrices and $P$ can be the same matrix as the one used in (25). Corresponding to this definition of $Z$ we will have $Z \bar{S}=$ $\bar{S} P^{-1}$. Substitute $Z \bar{S}=\bar{S} P^{-1}$ into (25) to get:

$\bar{S}^{*}\left(A Z+B K Z+Z A^{*}+Z K^{*} B^{*}+Z Q Z+Z K^{*} R K Z\right) \bar{S} \leq 0$

Introduce a new variable $W=K Z$, so that we have:

$\bar{S}^{*}\left(A Z+B W+Z A^{*}+W^{*} B^{*}+Z Q Z+W^{*} R W\right) \bar{S} \leq 0$

This can be written as an LMI condition using the Schur complement and noticing that $R>0$ and $Q \geq 0$, we get

$$
\begin{aligned}
& {\left[\begin{array}{lll}
\Upsilon & \bar{S}^{*} Z Q^{1 / 2} & \bar{S}^{*} W^{*} R^{1 / 2} \\
Q^{1 / 2} Z \bar{S} & -I & 0 \\
R^{1 / 2} W \bar{S} & 0 & -I
\end{array}\right] \leq 0,} \\
& \Upsilon=\bar{S}^{*}\left(A Z+B W+Z A^{*}+W^{*} B^{*}\right) \bar{S}
\end{aligned}
$$

Therefore, the minimization problem in (23) can be written as follows:

$$
\begin{aligned}
& \text { mintrace }(P) \text { s.t. } \\
& {\left[\begin{array}{lll}
\Upsilon & \bar{S}^{*} Z Q^{1 / 2} & \bar{S}^{*} W^{*} R^{1 / 2} \\
Q^{1 / 2} Z \bar{S} & -I & 0 \\
R^{1 / 2} W \bar{S} & 0 & -I
\end{array}\right] \leq 0} \\
& \Upsilon=\bar{S}^{*}\left(A Z+B W+Z A^{*}+W^{*} B^{*}\right) \bar{S}, \\
& Z=\bar{S} \bar{S}^{*} Z \bar{S} \bar{S}^{*}+S S^{*} Z S S^{*}
\end{aligned}
$$

where $Z \bar{S}=\bar{S} P^{-1}$. In the following we discuss the conditions for existence of a solution to the above minimization problem and then present the main results of this section as a theorem.

Discussion on the Existence of Solutions: It is well-known that detectability and stabilizability conditions are required for existence of a solution to a linear optimal control problem. The following lemma illustrates and formulates these conditions for our specific problem.

Lemma 2 The minimization problem (23), or equivalently (29), subject to the dynamical constraint (13) has an optimal stabilizing solution if matrices $A, B, Q$ are given such that the following inequalities have a solution for $P_{2}$ :

1. Stabilizability condition: $\bar{S}^{*}\left(A P_{2}+P_{2} A^{*}-B B^{*}\right) \bar{S}<0$,

2. Detectability condition: $\bar{S}^{*}\left(P_{2} A+A^{*} P_{2}-Q\right) \bar{S}<0$,

where $P_{2}>0$ satisfies $P_{2}=\bar{S} \bar{S}^{*} P_{2} \bar{S} \bar{S}^{*}+S S^{*} P_{2} S S^{*}$. 
Proof: Omitted due to space limitations.

The immediate result from the above discussions is the following theorem.

Theorem 3 a. Consider the dynamical system and the cost function given by (6) and (14), respectively. Assume that the matrices $A, B, Q$ satisfy the conditions of Lemma 2 . Moreover, assume that the control input $U$ is selected as $U=K X$, where $K=W Z^{-1}$ and the LMI variables $\Gamma, W$ and $Z$ are obtained through the minimization problem below

$$
\begin{aligned}
& \min \operatorname{trace}(\Gamma) \text { s.t. } \\
& \left\{\begin{array}{l}
\text { 1. }\left[\begin{array}{ll}
\Gamma & I \\
I & \bar{S}^{*} Z \bar{S}
\end{array}\right]>0 \\
\text { 2. }\left[\begin{array}{lll}
\Upsilon & \bar{S}^{*} Z Q^{1 / 2} & \bar{S}^{*} W^{*} R^{1 / 2} \\
Q^{1 / 2} Z \bar{S} & -I & 0 \\
R^{1 / 2} W \bar{S} & 0 & -I
\end{array}\right] \leq 0, \\
\Upsilon=\bar{S}^{*}\left(A Z+B W+Z A^{*}+W^{*} B^{*}\right) \bar{S}
\end{array}\right]=\left(\begin{array}{ll}
\text { 3. } & (A Z+B W) S=0 \\
\text { 4. } Z=\bar{S}^{*} Z \bar{S} \bar{S}^{*}+S S^{*} Z S S^{*}, Z>0
\end{array}\right.
\end{aligned}
$$

Then, the cost function (14) is minimized and the system described by (6) reaches a stable consensus in an optimal manner.

b. Furthermore, if the following constraints are added to the above minimization problem the controller would then be semi-decentralized. In other words, only partial information available through the predefined neighboring sets are used by the controller provided that:

$$
\left\{\begin{array}{l}
\text { 1. } Z \text { is diagonal, i.e. } Z=\left[\begin{array}{ccc}
Z_{1} & \ldots & 0 \\
\vdots & \ddots & \vdots \\
0 & \ldots & Z_{N}
\end{array}\right] \\
\text { 2. } W(i, j)=0 \text { if } L(i, j)=0
\end{array}\right.
$$

where $L$ is the Laplacian matrix of the graph describing the network.

Proof: Omitted due to space limitations.

\section{Discussion on Graph Connectivity}

So far there has been no explicit restriction on the connectivity of the network underlying graph. In the following we observe that the graph connectivity is a requirement for guaranteeing consensus achievement. First, we prove the following lemma which is required for the remainder of the discussion in this section.

Lemma 4 The closed-loop matrix of the entire network, i.e. $A+B K$ represents the Laplacian matrix of a weighted graph. The corresponding graph is a subgraph of the original network graph but with different weights assigned to its edges.

Proof: Omitted due to space limitations.

Theorem 5 If the graph corresponding to the entire network is not connected, the existence of a solution to the consensus problem cannot be guaranteed.
Proof: Omitted due to space limitations.

\section{Simulation Results}

It is worth noting that in the present approach, there is more flexibility in design of both the local controllers and the interaction terms. In other words, $u_{l}^{i}$ in (4) can be a function of both the local information $X^{i}$ as well as the global information $X^{j}, j \in N^{i}$. Consequently, the interaction term $F^{i j}$ can be selected as zero even though the agent $j$ is in the neighboring set of agent $i$.

Simulation results presented in this section are for a team of four agents. The simulations are done for two cases. In the first case the requirement given in Lemma 1 is not satisfied by the system matrix $A$, i.e. $A S \neq 0$. The Laplacian matrix corresponding to the connected graph describing the network structure is $L=\left(\begin{array}{cccc}2 & -1 & 0 & -1 \\ -1 & 2 & -1 & 0 \\ 0 & -1 & 2 & -1 \\ -1 & 0 & -1 & 2\end{array}\right)$. Other simulation parameters are $A^{i}=\left(\begin{array}{cc}1 & 1 \\ 0.5 & 1\end{array}\right), \quad B^{i}=2 I_{2 \times 2}, \quad c^{i}=$ $I_{2 \times 2}, \quad Q^{i j}=6 I_{2 \times 2}, \quad R^{i}=2 I_{2 \times 2}$. The state vector $X$ is composed of velocity vectors of all agents in the team, i.e. $X=\left[\left(v^{1}\right)^{T}, \ldots,\left(v^{N}\right)^{T}\right]^{T}$. Therefore, for the present case and assuming that the velocity vector is two-dimensional, i.e. $v^{i}=\left[\begin{array}{ll}v_{x}^{i}, & v_{y}^{i}\end{array}\right]^{T}$, the initial condition of the state vector is selected as $X(0)=\left(\begin{array}{llllllll}6 & 1 & 5 & 3 & 2 & 1 & -5 & -4\end{array}\right)^{T}$. The interaction coefficients $F^{i j}, \forall i, j$ are assumed to be zero. It can be verified that the above parameters satisfy the conditions provided in Lemma 2 and the corresponding matrix $P_{2}$ can be selected as the identity matrix. The simulation results are obtained by applying the control law $U=K X$ to the system (6). Matrix $K$ is first evaluated through the set of LMIs given in Theorem 3. In Figure 1, the $x$-component, i.e. $v_{x}^{i}$ of the velocity profiles of the four-agent team are shown for the above configuration. In Figure 2 we have applied the control strategy given by (17) to the system (6) with the above configuration. It can be seen that as predicted in Lemma 1 the closed-loop system is unstable. This is due to the fact that the open-loop matrix $A$ does not satisfy the property $A S=0$.

In the second part of the simulations, we have selected a matrix $A$ such that $A S=0$. The corresponding results are presented in Figures 3-4. To guarantee the condition $A S=0$, the interaction coefficients are selected as $F^{i j}=$ $-0.25\left(\begin{array}{cc}1 & 1 \\ 0.5 & 1\end{array}\right), \forall i, j$. Other simulation parameters are the same as in the previous case. The simulation results are obtained by applying the controllers designed based on the Riccati equation solutions and the solutions to the set of LMIs in (30). Figure 3 corresponds to the latter approach, whereas Figure 4 corresponds to the former approach. For comparison between these two approaches we calculated a performance index for both methods. Since one does not have direct access to $X_{\bar{s}}$, instead of the performance index (14) we used the following cost function for comparative 
purposes:

$$
P I=\int_{0}^{T}\left\{X^{T} Q X+U^{T} R U\right\} d t
$$

where $T$ is selected to be $10 \mathrm{~s}$. The values obtained for the above performance index are 865.5 and 883.2 corresponding to the Riccati equation and the LMIs approaches, respectively.

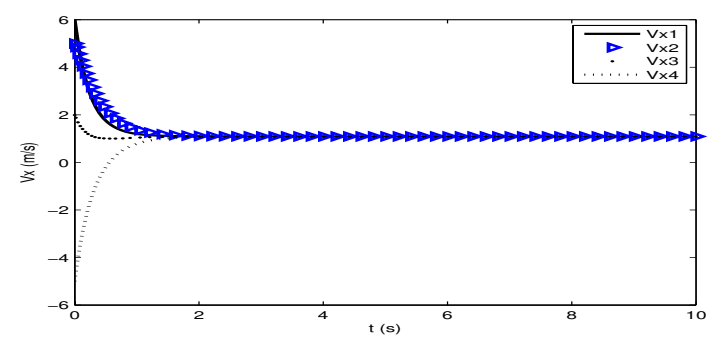

Fig. 1. The $x$-component of the velocity profile; optimal design based on the solution of the LMIs when $A S \neq 0$.

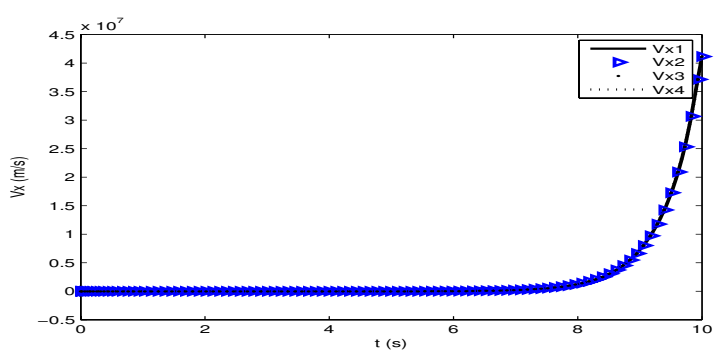

Fig. 2. The $x$-component of the velocity profile; optimal design based on the solution of the Riccati equation when $A S \neq 0$.

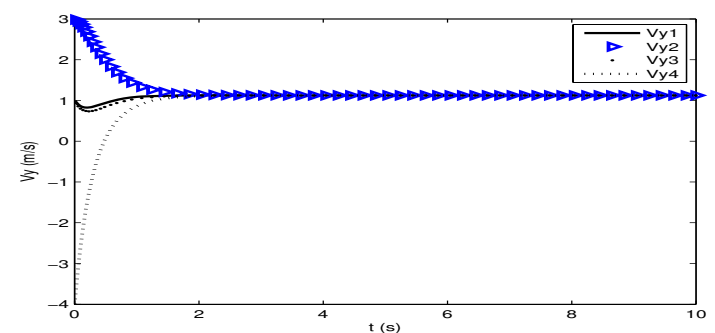

Fig. 3. The $y$-component of the velocity profile; optimal design based on the solution of the LMIs when $A S=0$.

\section{CONCLUSIONS}

An optimal control design strategy based on state decomposition is introduced to guarantee consensus achievement in a network of multi-agents. It is shown that the approach based on the solution of the Riccati equation in general fails to provide a solution for a stable consensus protocol. Therefore, our proposed approach is based on minimization of a global cost function that is subject to a set of constraints expressed as LMIs. Introduction of a global cost function considered here can ensure a global optimal (or suboptimal) solution. Also, this formulation provides a single index for describing and analyzing the total performance of the team.

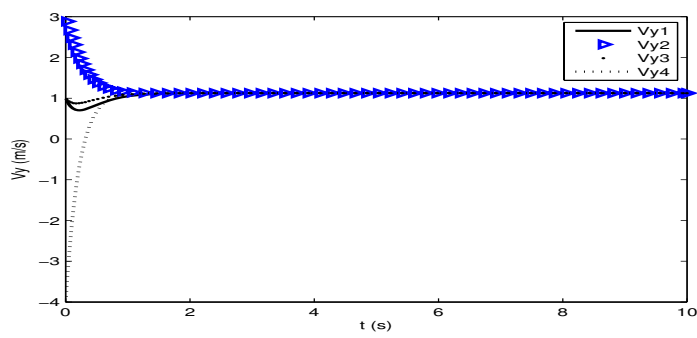

Fig. 4. The $y$-component of the velocity profile; optimal design based on the solution of the Riccati equation when $A S=0$.

Moreover, through the LMI formulation the constraint on partial information availability can be formally taken into account. Therefore, in the individual control design the only required information will be what has been received from the corresponding neighbors in the controller's neighboring set. This framework has sufficient flexibility to accommodate additional constraints and design criteria in our proposed methodology and solution.

\section{REFERENCES}

[1] R. W. Beard, J. Lawton, and F. Y. Hadaegh, "A coordination architecture for spacecraft formation control," IEEE Trans. Contr. Syst. Technol., vol. 9, no. 6, pp. 777-789, 2001.

[2] B. Sinopoli, C. Sharp, L. Schenato, S. Schafferthim, and S. Sastry, "Distributed control applications within sensor networks," Proc. IEEE, vol. 91, no. 8, pp. 1235-1246, 2003.

[3] D. Lee and M. W. spong, "Stable flocking of inertial agents on balanced communication graphs," IEEE Trans. Automat. Contr., vol. 52, no. 8, pp. 1469-1475, 2007.

[4] W. Li, "Stability analysis of swarms with general topology," IEEE Trans. Syst., Man, Cybern. B, vol. 38, no. 4, pp. 1084-1097, 2008.

[5] F. Kunwar and B. Benhabib, "Rendezvous-guidance trajectory planning for robotic dynamic obstacle avoidance and interception," IEEE Trans. Syst., Man, Cybern. B, vol. 36, no. 6, pp. 1432-1441, 2006.

[6] W. Ren and R. W. Beard, "Formation feedback control for multiple spacecraft via virtual structures," IEE proc. control theory appl., vol. 151, no. 3, pp. 357-368, 2004.

[7] R. Olfati-Saber and R. M. Murray, "Consensus problems in networks of agents with switching topology and time-delays," IEEE Trans. Automat. Contr., vol. 49, no. 9, pp. 1520-1533, 2004.

[8] F. Xiao and L. Wang, "Consensus problems for high-dimensional multi-agent systems," IET Control Theory Applications, vol. 1, no. 3, pp. 830-837, 2007.

[9] W. Ren and R. W. Beard, "Consensus seeking in multiagent systems under dynamically changing interaction topologies," IEEE Trans. Automat. Contr., vol. 50, no. 5, pp. 655-661, 2005.

[10] M. Arcak, "Passivity as a design tool for group coordination," IEEE Trans. Automat. Contr., vol. 52, no. 8, pp. 1380-1390, 2007.

[11] D. Bauso, L. Giarre, and R. Pesenti, "Mechanism design for optimal consensus problems," in Proc. Conference on Desision and Control, Dec. 13-15, 2006, pp. 3381-3386.

[12] E. Semsar and K. Khorasani, "Optimal control and game theoretic approaches to cooperative control of a team of multi-vehicle unmanned systems," in Proc. IEEE International Conference on Networking, Sensing and Control, Apr. 15-17, 2007, pp. 628-633.

[13] E. Semsar-Kazerooni and K. Khorasani, "Optimal consensus algorithms for cooperative team of agents subject to partial information," Automatica, vol. 44, no. 11, pp. 2766-2777, 2008.

[14] G. A. Decastro and F. Paganini, "Convex synthesis of controllers for consensus," in Proc. American Control Conference, June 30-July 2, 2004, pp. 4933-4938.

[15] S. Boyd, L. E. Ghaoui, E. Feron, and V. Balakrishnan, Linear Matrix Inequalities in System and Control Theory. SIAM, 1994.

[16] A. Jadbabaie, "Robust, non-fragile controller synthesis using modelbased fuzzy systems: A linear matrix inequality approach," Master's thesis, The University of New Mexico, 1997. 